\title{
The Color and Blink Frequency of LED Notification Lights and Smartphone Users' Urgency Perception
}

\author{
Minsun Kim ${ }^{1}$, Jiyeun Lee ${ }^{1}$, Hyebeen Lee ${ }^{1}$, Soyeon Kim ${ }^{1}$, \\ Haemi Jung ${ }^{2}$, and Kwanghee Han ${ }^{1}$, \\ ${ }^{1}$ Cognitive Engineering Lab. Department of Psychology, Yonsei University, Korea \\ kimmin0414@hanmail.net, amethystljy@naver.com, \\ hblee27@gmail.com, england311@naver.com, khan@yonsei.ac.kr \\ ${ }^{2}$ UX Lab, Mobile R\&D Center, LG Electronics, Korea \\ hyemi.jungalge.com
}

\begin{abstract}
LED notification lights are used in smartphones to deliver various information to users, such as missed calls, incoming calls, receiving a new text message, and low battery. This study explores whether the settings of the color and blink frequency of the LEDs notification lights affect smartphone users' urgency perception in two important information states, i.e., missed calls and incoming calls. We set the blink frequency of LED notification lights high and low, assuming that high frequency blink will be perceived as more urgent notification. As for color, we first set the color of LED indicators as White for both high and low blink frequencies. Then, we changed the color to Red for high frequency blink and Green for low frequency blink to examine whether color has any effect on smartphone users' urgency perception of the notification. We measured users' urgency perception by asking participants to evaluate how effective each setting of LED notification lights is in indicating the degree of urgency of the corresponding information state. In an experiment with 30 participants, we found that participants perceived high blink frequency as more intuitive and attractive and therefore more effective than low blink frequency in indicating high urgency in both information states. We also found that the use of color increases users' overall urgency perception. We further found that users' urgency perception-both intuitive and attentive-was highest when color was used and the blink rate of notification was high. That is, the urgency perception score was highest when the color was Red and the blink frequency was high. In the post-experiment interviews, participants chose red as the best color to indicate urgency. Given that, we conclude that the use of red color with high blink frequency of the LED notification light is most effective in conveying urgency information.
\end{abstract}

Keywords: LED notification light, smartphone, urgency perception, color, blink frequency.

* Corresponding author. 


\section{Introduction}

The smartphone LED notification lights help users to recognize various situations without activating the display. The LED notification lights use movement pattern and color to deliver various information states to users. For instance, movement pattern of lighting can deliver different information states to users such as warning or progressing by using different blink frequencies. Color is also useful in providing specific information because people have specific preconceived images of particular colors. Therefore, when a color is used in a way that does not match users' preconceived image of the color, intended information cannot be effectively delivered (Kang, 2006).

This study examines whether the use of movement pattern and color of smartphone LED notification lights can deliver intended information more effectively when they match users' mental model. This study focuses on two emergency situations, i.e., notification of missed calls and incoming calls, where LED notification is most frequently used. Specifically, we examine whether users perceive the urgency of the situations differently - in both cognitive and emotional aspects-as the blink frequency and color of LED lighting change. Perceived urgency leads individuals to take immediate actions or attentions to the situation (Suied, Susini, \& McAdams, 2008) because individuals respond more appropriately and promptly to the situations when they perceive greater urgency of the situations (Guillaume et al., 2003; Bliss, Gilson, \& Deaton, 1955).

Prior studies on colors found that long wavelength colors such as red have a greater arousing effect than short wavelength colors such as blue or green (Elliot, Maier, Moller, Friedman, \& Meinhardt, 2007). Because colors with greater arousing effect can draw more attention from individuals, we predict that red color LED lighting will be more effective in generating perceived urgency among smartphone users. We also predict that smartphone users will perceive greater urgency when high blink frequency is used than when low blink frequency is used. We further predict that when both red color and high blink frequency are used together, smartphone users will perceive greater urgency than when they are separately used.

\section{Experiment}

\subsection{Participants}

30 undergraduate students participated in this study. All participants had normal vision and all of them were smartphone users.

\subsection{Experimental Design}

This study was conducted to verify the effect of blink frequency (speed) and pattern's color on perceived urgency. Blink frequency (high, low) and color (without color, color) were manipulated to compare the perceived urgency of Smartphone LED. It was 2 X 2 within factorial experimental design. 


\subsection{Experimental Tasks and Procedures}

Before the experiment conducted, participants received Smartphone samples that would be used for the experiment. Then they turned on the several LED themselves in order to understand the experiment well. The LED which participants had to observe during the test was situated on the top of front of Smartphone. After simple instruction, participants took part in the experiment. The experiment was proceeded with the sample smartphone's display was covered. Participants were given two situations and there were four patterns in each situation. In a concrete aspect, the situations were consisted of incoming calls and notification of missed calls. Four smartphone LED patterns were presented from the combination of frequency (high, low) and color (without color, color) in each situation (table1).

Condition of frequency was consisted of high and low blinking conditions of LED light in identical time. Because the high frequency condition blinked more in the same amount of time, participants perceived the speed faster than low frequency one. We labeled the high blinking condition as urgent condition, and low blinking condition as normal condition. For the color condition, white color was presented as condition of without color (white). With color condition, normal condition was presented with green and urgent condition was presented with red to classify.

Table 1. Four LED types in each situation

\begin{tabular}{|c|c|c|c|c|}
\hline Situation & \multicolumn{2}{|c|}{ 1. Missed Call } & \multicolumn{2}{c|}{ 2. Incoming Call } \\
\hline \multirow{2}{*}{ no color } & $\begin{array}{c}\text { (Normal) } \\
\text { Low Frequency }\end{array}$ & $\begin{array}{c}\text { (Urgent) } \\
\text { High Frequency }\end{array}$ & $\begin{array}{c}\text { (Normal) } \\
\text { Low Frequency }\end{array}$ & $\begin{array}{c}\text { (Urgent) } \\
\text { High Frequency }\end{array}$ \\
\hline \multirow{2}{*}{ color } & $\begin{array}{c}\text { Pattern 1 } \\
\text { (White) }\end{array}$ & $\begin{array}{c}\text { Pattern 2 } \\
\text { (White) }\end{array}$ & $\begin{array}{c}\text { Pattern 1 } \\
\text { (White) }\end{array}$ & $\begin{array}{c}\text { Pattern 2 } \\
\text { (White) }\end{array}$ \\
\hline $\begin{array}{c}\text { Pattern 3 } \\
\text { (Green) }\end{array}$ & $\begin{array}{c}\text { Pattern 4 } \\
\text { (Red) }\end{array}$ & $\begin{array}{c}\text { Pattern 3 } \\
\text { (Green) }\end{array}$ & $\begin{array}{c}\text { Pattern 4 } \\
\text { (Red) }\end{array}$ \\
\hline
\end{tabular}

The task for the participants was to assess how each pattern delivered urgent meaning effectively in cognitive and emotional aspects by comparing the conditions of changing frequency and changing both color and frequency. As for the questionnaire, intuitiveness (Current situation can be noticed through LED) and attentiveness (LED catches eyes and outstands) were presented for cognitive aspect of items, each item was measured with 7-point likert scale.

As for emotional aspect, emotional vocabularies which were related to urgency were selected. Participants measured how the given patterns match well with each vocabulary. In a detailed aspect, adjectives relate to urgency presented on two axes, and then participants had to situate the patterns on a spot on each axis. The adjectives related to urgency such as 'important', 'urgent' were chosen from the previous research. After finishing questionnaire for every situation and pattern, simple instruction of the experiment was presented and the experiment ended. 


\section{$3 \quad$ Result}

The result is analyzed by comparing the measurement of intuitiveness and attentiveness in cognitive aspect, and by emotional words in the emotional aspect through SPSS 18.0 statistical program.

Firstly urgency perception depending on the frequency of pattern (urgent/normal) is analyzed by t-test, and there is a significant difference in cognitive aspect in without color condition, Missed call: $\mathrm{t}(29)=-2.576, \mathrm{p}=.015$ (Intuitiveness), $\mathrm{t}(29)=-3.294$, $\mathrm{p}=.003$ (Attentiveness); Incoming call: $\mathrm{t}(29)=-2.567, \mathrm{p}=.016$. In color condition, there is also a significant difference, Missed call: $\mathrm{t}(29)=-9.485, \mathrm{p}=.001$ (Intuitiveness), $\mathrm{t}$ (29) $=-6.747, \mathrm{p}=.001$ (Attentiveness); Incoming call: $\mathrm{t}(29)=-7.099$, $\mathrm{p}=.001$ (Intuitiveness), $\mathrm{t}(29)=-4.878, \mathrm{p}=.001$ (Attentiveness). It is higher score of attentiveness and intuitiveness in urgent condition than in normal condition. Also in emotional aspect urgent condition shows higher score than normal condition, in without color condition, Missed call: $\mathrm{t}(29)=-9.549, \mathrm{p}=0.001$ (Urgent), $\mathrm{t}(29)=-10.476$, $\mathrm{p}=.001$ (Important); Incoming call: $\mathrm{t}(29)=-9.492, \quad \mathrm{p}=.001$ (Urgent), $\mathrm{t}(29)=-7.94$, $\mathrm{p}=.001$ (Important). In color condition, there is also a significant difference, Missed call: $\mathrm{t}(29)=-4.36, \mathrm{p}=.001$ (Urgent), $\mathrm{t}(29)=-2.677, \mathrm{p}=.012$ (Important); Incoming call: $\mathrm{t}(29)=-2.904, \mathrm{p}=.007$ (Urgent), $\mathrm{t}(29)=-3.188, \mathrm{p}=.003$ (Important) .

In addition, urgency perception depending on color is analyzed by ANOVA. There is a significant difference, Missed call: $\mathrm{F}(1,29)=20.39, \mathrm{p}<.001$ (Intuitiveness), $\mathrm{F}(1,29)=13.08, \mathrm{p}<.001$ (Attentiveness); Incoming call: $\mathrm{F}(1,29)=11.868, \mathrm{p}<.05$ (Intuitiveness), $\mathrm{F}(1,29)=13.67, \quad \mathrm{p}=.001$ (Attentiveness). Users' urgency perception-both intuitiveness and attentiveness- is the highest when color is used and the blink rate of notification is high. The urgency perception score is the highest when the color is Red and the blink frequency is high. There is similar result from emotional aspect. In the condition of with color, it is higher score than in the condition without color when measure of emotional words including urgent and important, Missed call: $F(1,29)=33.143$, $\mathrm{p}<.001$ (Urgent), $\mathrm{F}(1,29)=29.876, \mathrm{p}<.001$ (Important); Incoming call: $\mathrm{F}(1,29)=17.939$, $\mathrm{p}<.001$ (Urgent), $\mathrm{F}(1,29)=11.744, \mathrm{p}<.05$ (Important). Furthermore, it is similar with the result of cognitive aspect that in the emotional aspect, the conditions of red and high blink frequency makes the highest score.
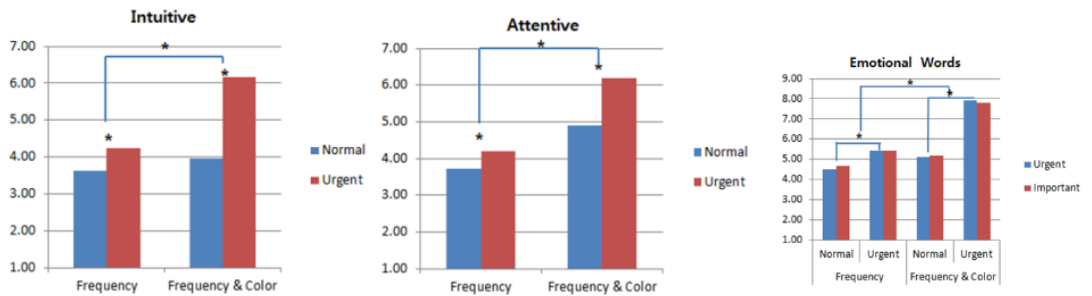

Fig. 1. Urgent perception score in Cognitive aspect (Left, Middle) and Emotional aspect (Right) of missed call 

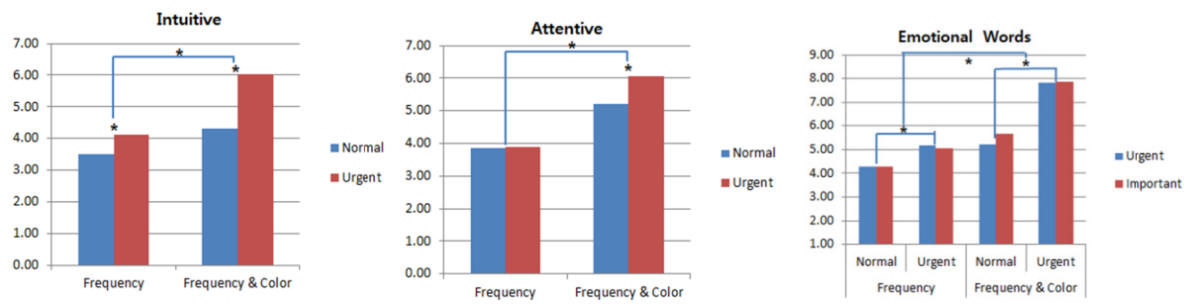

Fig. 2. Urgent Perception score in Cognitive aspect (Left, Middle) and Emotional aspect (Right) of incoming call

\section{Conclusion}

The present study showed that how the movement pattern and color of smartphone LED influenced users' urgency perception. As a result of measuring perceived urgency by manipulating LED blink frequency and color of smartphone, it is identified that high frequency pattern make meaningful higher score in cognitive aspect and emotional aspect and that the difference can be increased with using color. Also qualitative analysis of users through post-experiment interviews shows that most participants chose red as the best color to indicate urgency. Given that, we conclude that the use of red color with high blink frequency of the LED notification light is most effective in conveying urgency information. As stated earlier, people perceived urgency from red because a long wavelength color such as red has a greater arousing effect. To conclude, this study makes an important contribution by demonstrating that smartphone LED notification lights can be used to effectively deliver important messages to users when the movement pattern and color of LED lightings are properly designed.

Acknowledgement. This work has been supported by UX Lab, Mobile R\&D Center, LG Electronics.

\section{References}

1. Bliss, J.P., Gilson, R.D., Deaton, J.E.: Human probability matching behavior in response to alarms of varying reliability. Ergonomics 38, 2300-2312 (1995)

2. Elliot, A.J., Maier, M.A., Moller, A.C., Friedman, R., Meinhardt, J.: Color and psychological functioning: the effect of red on performance attainment. Journal of Experimental Psychology: General 136, 154-168 (2007)

3. Guillaume, A., Pellieux, L., Chastres, V., Drake, C.: Judging the urgency of nonvocal auditory warning signals: Perceptual and cognitive processes. Journal of Experimental Psychology: Applied 9, 196-212 (2003)

4. Suied, C., Susini, P., McAdams, S.: Evaluating warning sound urgency with reaction times. Journal of Experimental Psychology: Applied 14, 201-212 (2008)

5. Kang, S.J.: The Functions of Color Based on Human Cognition in Information Design. Journal of Korean Society of Color Studies 20, 1-10 (2011) 